\title{
Direct Bilirubin in Drainage Fluid 3 days after Hepatectomy Is Useful for Detecting Severe Bile Leakage
}

\author{
Norifumi Harimoto ${ }^{a}$ Kenichiro Araki ${ }^{a}$ Takahiro Yamanaka ${ }^{a}$ Kei Hagiwara ${ }^{a}$ \\ Norihiro Ishii ${ }^{a}$ Mariko Tsukagoshi ${ }^{b}$ Akira Watanabe ${ }^{a}$ Ken Shirabe ${ }^{a}$ \\ aDivision of Hepatobiliary and Pancreatic Surgery, Department of General Surgical Science, Graduate School of \\ Medicine, Gunma University, Maebashi, Japan; bepartment of Innovative Cancer Immunotherapy, Graduate \\ School of Medicine, Gunma University, Maebashi, Japan
}

\section{Keywords}

Bile leakage $\cdot$ Hepatectomy $\cdot$ Drain fluid $\cdot$ Direct bilirubin

\begin{abstract}
Introduction: The International Study Group of Liver Surgery (ISGLS) definition of bile leakage is an elevated total bilirubin concentration in the drainage fluid after post-operative day (POD) 3, which has been widely accepted. However, there were no reports about direct bilirubin in drainage fluid to predict bile leakage. Methods: Data from 257 patients who underwent hepatectomy were retrospectively reviewed. The optimal cut-off value was investigated using receiveroperating characteristic curves. The predictive power of drainage fluid total bilirubin (dTB) and drainage fluid direct bilirubin (dDB) to predict bile leakage, which was defined using ISGLS grade B or grade C, were compared. Results: ISGLS grade $B$ bile leakage occurred in 16 patients (6.2\%). Area under the curve (AUC) of dDB was always higher than that of dTB on each POD. The AUC of dDB was $>0.75$ on PODs 2, 3, and 5 , and then it increased with the increasing POD. The dDB on POD 5 showed the highest accuracy (0.91) and positive predictive value (PPV) (0.67), which was followed by $\mathrm{dTB}$ /serum total bilirubin (sTB) on POD 3 (accuracy, 0.91; PPV, 0.33). Because the PPV of $d D B$ increased as the POD in-
\end{abstract}

creased, dDB was better than dTB for predicting clinically significant bile leakage. dDB on POD 3 showed the highest negative predictive value (0.97). The positive likelihood of $\mathrm{dDB}$ increased and the negative likelihood of $\mathrm{dDB}$ decreased on the basis of the POD. Among patients with $d T B / s T B \leq 3$ on POD 3, $19.1 \%$ of these patients had bile leakage when dDB was $>0.44$ on POD 3. Conclusions: Measurement of both dDB and dTB, which are easy to perform, can be used to effectively predict clinically significant bile leakage.

(c) 2021 S. Karger AG, Basel

\section{Introduction}

Hepatectomy can be a risky procedure, but strict evaluation of liver function and improvements to the perioperative management have led to low mortality and morbidity rates $[1,2]$. Bile leakage is the most common postoperative complication and cause of morbidity. We previously showed that clinically significant bile leakage occurred in $4.4 \%$ of surgically treated patients, and poor liver function, hepatocellular carcinoma, and high-risk procedures were independent risk factors for bile leakage after hepatectomy [3]. karger@karger.com www.karger.com/esr
(C) 2021 S. Karger AG, Basel 
Clinically significant bile leakage is determined on the basis of bile accumulation in abdominal fluid, fever, and increased inflammatory response if there is no drain. Thus, an abdominal drain is placed to detect abnormal fluid at an early stage, but sometimes it is not effective. Thus, it is often removed, and clinically significant bile leakage becomes apparent later. Percutaneous drainage is risky and stressful for both the healthcare professionals and patients. Thus, detecting clinically significant bile leakage at an early stage is useful.

The International Study Group of Liver Surgery (ISGLS) definition of bile leakage has been widely used to grade bile leakage and determine its severity $[4,5]$. An elevated total bilirubin concentration in the drainage fluid after post-operative day (POD) 3 is important; however, whether direct bilirubin is useful in predicting bile leakage is unclear. Total bilirubin is the sum of direct and indirect bilirubin, and total bilirubin levels, but not direct bilirubin, in the drainage fluid increase when haemolysis occurs at the cut surface of the liver. Indirect bilirubin levels increase when haemolysis occurs, which may explain why total bilirubin levels in drainage fluids increase without bile leakage. However, direct bilirubin, which is conjugated bilirubin, originates from hepatocytes. Thus, our hypothesis in this study was that direct bilirubin levels in drainage fluids may be useful to predict bile leakage after hepatectomy.

This retrospective study was conducted on the basis of these clinical questions, and it was performed to investigate the significance of direct bilirubin in the drainage fluid as a predictor of clinically significant bile leakage after hepatectomy in patients with hepatic malignancy.

\section{Materials and Methods}

\section{Patient Population}

Data on the total and direct bilirubin levels in the drainage fluid from 257 patients who underwent hepatectomy for hepatic malignancy at the Division of Hepatobiliary and Pancreatic Surgery, Department of General Surgical Science, Gunma University from November 2018 to March 2021 were retrospectively collected. The study protocol was approved by our institutional Ethics Committee (Gunma University Hospital Clinical Research Review Board, approval number: HS2019-306). Patients who underwent hepatectomy with biliary reconstruction were excluded.

Clinically significant bile leakage was defined as ISGLS grade B or C bile leakage. The ISGLS definition of bile leakage is defined as follows [4]: drainage of fluid with a total bilirubin level 3-times greater than the serum total bilirubin (sTB) level on or after POD 3 or the need for interventions as the result of bile collection. Bile leakage requiring little or no change in the patient's clinical management was classified as Grade $\mathrm{A}$, and bile leakage requiring a change in the patient's clinical management (e.g., additional diagnostic or interventional procedures) but manageable without relaparotomy was considered to be Grade B. A grade A bile leakage lasting for $>1$ week was also considered to be Grade B. Grade C was defined as bile leakage that required relaparotomy.

The albumin-indocyanine green evaluation (ALICE) grade was calculated using the indocyanine green retention rate at $15 \mathrm{~min}$ (ICGR15) and the serum albumin level. The formula is as follows: $0.663 \times \log 10$ ICGR15-0.718 $\times$ albumin $(\mathrm{g} / \mathrm{dL})[6]$. The ALICE grade was classified as follows: ALICE grade $1(<-2.20)$, ALICE grade $2(-2.20$ to -1.39$)$, and ALICE grade 3 (>-1.39). A high-risk procedure was defined as more frequent exposure of the Glissonean sheath such as central bi-segmentectomy and anterior segmentectomy or thrombectomy for a bile duct tumour thrombus [3]. Patients with diabetes were defined as those using insulin or taking oral medication.

\section{Operative Indication and Surgical Procedures}

Operative indication and surgical procedure details were reported previously $[3,7,8]$. Hepatectomy candidate patients had no ascites or controllable ascites with diuretics. Liver volume was calculated using computed tomography and EOB-magnetic resonance imaging, and the future remnant liver volume was $>615 \mathrm{~mL} /$ $\mathrm{m}^{2}$ after removal of $>1$ segment [9]. Liver parenchymal transection was performed with an ultrasonic dissector and a Cavitron Ultrasonic Surgical Aspirator (AMCO Inc., Tokyo, Japan) using the Pringle manoeuvre [10]. All sizable vessels were ligated. Fibrin glue was routinely applied to the cut surface of the liver. A bile leakage test was usually performed after hepatectomy. The indication for cystic duct tube (c-tube) insertion was if patients required repeated repair for intraoperative bile leakage. An abdominal drain was routinely placed up to the cut edge of the liver and removed by POD 3 unless the drainage fluid showed large amounts of blood, exceeding $100 \mathrm{~mL} /$ day or if it did not meet the ISGLS bile leakage criteria.

\section{Statistical Analysis}

The statistical analyses were performed using the Wilcoxon rank sum test for continuous variables and the $\chi^{2}$ test for categorical variables. The best cut-off value of drainage fluid total bilirubin $(\mathrm{dTB})$ and drainage fluid direct bilirubin $(\mathrm{dDB})$ was investigated using receiver-operating characteristic (ROC) curves, and the area under the curve (AUC) was calculated. Statistical significance was defined as $p<0.05$. All analyses were performed using JMP version 11 software (SAS Institute, Inc., Cary, NC, USA).

\section{Results}

No hospital deaths occurred. Among the entire cohort $(n=257)$, bile leakage occurred in 21 patients $(8.2 \%)$ as follows: $5(1.9 \%)$ with ISGLS grade A leakage and 16 (6.2\%) with ISGLS grade B leakage. There were no reoperations. There were no patients with late-onset (over 2 weeks after surgery) bile leakage. Percutaneous abscess drainage was performed for 3 patients because the drain had already been removed when the bile leak was found. 
Table 1. Comparison of the clinicopathological factors between the 2 groups classified by clinically significant bile leakage

\begin{tabular}{|c|c|c|c|}
\hline \multirow{2}{*}{$\begin{array}{l}\text { Variables } \\
\text { Host-related factor }\end{array}$} & \multirow[t]{2}{*}{$\begin{array}{l}\text { Clinically significant } \\
\text { bile leakage }(-) \\
(n=241)\end{array}$} & \multicolumn{2}{|c|}{$\begin{array}{l}\text { Clinically significant } p \text { value } \\
\text { bile leakage }(+) \\
(n=16)\end{array}$} \\
\hline & & & \\
\hline Age & $67.5 \pm 12.0$ & $72.7 \pm 7.6$ & 0.09 \\
\hline Male/female & $163 / 78$ & $14 / 2$ & 0.16 \\
\hline $\mathrm{BMI}, \mathrm{kg} / \mathrm{m}^{2}$ & $24.3 \pm 13.0$ & $22.8 \pm 2.8$ & 0.65 \\
\hline $\mathrm{HBV}, n(\%)$ & $50(20.7)$ & $4(25.0)$ & 0.44 \\
\hline $\mathrm{HCV}, n(\%)$ & $70(29.0)$ & $6(37.5)$ & 0.32 \\
\hline Diabetes mellitus, $n(\%)$ & $65(27.0)$ & $5(31.3)$ & 0.45 \\
\hline Albumin, g/dL & $4.1 \pm 0.4$ & $3.9 \pm 0.4$ & 0.02 \\
\hline Total bilirubin, mg/dL & $0.8 \pm 0.3$ & $0.9 \pm 0.4$ & 0.08 \\
\hline Platelet count, $10^{4} / \mu \mathrm{L}$ & $19.8 \pm 7.0$ & $17.8 \pm 5.0$ & 0.26 \\
\hline ICGR15 (\%) & $13.3 \pm 8.2$ & $18.5 \pm 6.9$ & 0.01 \\
\hline Child-Pugh A/B & $239 / 2$ & $16 / 0$ & 0.99 \\
\hline ALICE $1 / 2$ or 3 & $148 / 93$ & $5 / 11$ & 0.03 \\
\hline Fibrosis $(\mathrm{F} 0+1+2 / 3+4)$ & $156 / 85$ & $9 / 7$ & 0.33 \\
\hline \multicolumn{4}{|l|}{ Tumour-related factor } \\
\hline HCC/non-HCC & $95 / 146$ & $12 / 4$ & $<0.01$ \\
\hline \multicolumn{4}{|l|}{ Surgical factor } \\
\hline Operation time, $\min$ & $336 \pm 103$ & $386 \pm 65$ & 0.06 \\
\hline Blood loss, $\mathrm{g}$ & $178 \pm 239$ & $563 \pm 852$ & $<0.01$ \\
\hline Transfusion (+), $n(\%)$ & $8(3.3)$ & $1(6.3)$ & 0.44 \\
\hline Anatomical/non-anatomical & $105 / 136$ & $14 / 2$ & $<0.01$ \\
\hline High-risk procedure, $n(\%)$ & $18(7.5)$ & $8(50.0)$ & $<0.01$ \\
\hline C-tube insertion, $n(\%)$ & $32(13.3)$ & $4(25.0)$ & 0.25 \\
\hline Hospital stay, day & $11.9 \pm 37.7$ & $48.7 \pm 33.6$ & $<0.01$ \\
\hline Duration of drainage, day & $5.8 \pm 4.7$ & $45.3 \pm 26.6$ & $<0.01$ \\
\hline sTB (1POD) & $1.2(0.2-3.8)$ & $1.5(0.7-3.2)$ & 0.04 \\
\hline sTB (2POD) & $1.2(0.07-5.2)$ & $1.5(0.7-2.6)$ & 0.18 \\
\hline sTB (3POD) & $1.3(0.4-7.1)$ & $1.4(0.8-2.1)$ & 0.63 \\
\hline sTB (5POD) & $1.2(0.4-12.9)$ & $1.5(0.6-2.0)$ & 0.89 \\
\hline \multicolumn{4}{|l|}{ Drain factor } \\
\hline $\mathrm{dTB} / \mathrm{sTB}>3(3 \mathrm{POD})(n=241)(\%)$ & $10(4.4)$ & $5(31.3)$ & $<0.01$ \\
\hline $\mathrm{dTB}(1 \mathrm{POD}), \mathrm{mg} / \mathrm{dL}$ & $0.97 \pm 0.52$ & $3.68 \pm 10.12$ & $<0.01$ \\
\hline $\mathrm{dTB}(2 \mathrm{POD}), \mathrm{mg} / \mathrm{dL}$ & $1.28 \pm 0.62$ & $9.13 \pm 21.97$ & $<0.01$ \\
\hline $\mathrm{dTB}(3 \mathrm{POD}), \mathrm{mg} / \mathrm{dL}(n=241)$ & $1.87 \pm 0.93$ & $8.96 \pm 22.62$ & $<0.01$ \\
\hline $\mathrm{dTB}(5 \mathrm{POD}), \mathrm{mg} / \mathrm{dL}(n=157)$ & $2.61 \pm 1.38$ & $4.80 \pm 5.54$ & $<0.01$ \\
\hline $\mathrm{dDB}(1 \mathrm{POD}), \mathrm{mg} / \mathrm{dL}$ & $0.25 \pm 0.36$ & $2.57 \pm 8.68$ & $<0.01$ \\
\hline $\mathrm{dDB}(2 \mathrm{POD}), \mathrm{mg} / \mathrm{dL}$ & $0.21 \pm 0.33$ & $7.13 \pm 19.78$ & $<0.01$ \\
\hline $\mathrm{dDB}(3 \mathrm{POD}), \mathrm{mg} / \mathrm{dL}(n=241)$ & $0.21 \pm 0.36$ & $7.12 \pm 21.97$ & $<0.01$ \\
\hline $\mathrm{dDB}(5 \mathrm{POD}), \mathrm{mg} / \mathrm{dL}(n=157)$ & $0.27 \pm 0.39$ & $2.77 \pm 5.34$ & $<0.01$ \\
\hline
\end{tabular}

Data are expressed as means \pm standard deviations or number of patients (percentage) as appropriate. sTB are expressed as means (minimum, maximum). HBV, hepatitis B antigen positive; HCV, hepatitis $\mathrm{C}$ antibody positive; ICGR15, indocyanine green dye retension test at $15 \mathrm{~min}$; ALICE, albumin-indocyanine green evaluation; HCC, hepatocellular carcinoma; dTB, total bilirubin level in drainage fluid; sTB, serum total bilirubin level; $\mathrm{dDB}$, direct bilirubin level in drainage fluid; POD, post-operative day; BMI, body mass index.
Endoscopic naso-biliary drainage was required for 4 patients. Nine patients needed a drain exchange.

The relevant clinicopathological characteristics of patients with and without clinically significant bile leakage are shown in Table 1. Patients with clinically significant bile leakage had lower albumin levels, higher ICGR15 scores, an ALICE score of 2 or 3, a much higher hepatocellular carcinoma incidence, greater blood loss, anatomical resection, and more high-risk procedures than patients without clinically significant bile leakage. The 
Table 2. Predictive power of drainage factors for clinically significant bile leakage

\begin{tabular}{lllr}
\hline Variables, mg/dL & Cut-off value & AUC & $p$ value \\
\hline dTB (1POD) & 0.90 & 0.654 & 0.21 \\
dTB (2POD) & 1.60 & 0.714 & $<0.01$ \\
dTB (3POD) & 2.10 & 0.664 & $<0.01$ \\
dTB (5POD) & 4.10 & 0.655 & 0.03 \\
dDB (1POD) & 0.34 & 0.699 & $<0.01$ \\
dDB (2POD) & 0.21 & 0.784 & $<0.01$ \\
dDB (3POD) & 0.44 & 0.794 & $<0.01$ \\
dDB (5POD) & 1.35 & 0.809 & $<0.01$ \\
\hline
\end{tabular}

AUC, area under the curve; dTB, total bilirubin level in drainage fluid; $\mathrm{dDB}$, direct bilirubin level in drainage fluid; POD, postoperative day.

length of hospital stay and duration of drainage were significantly longer in patients with bile leakage than in those without. The incidence of 3-times higher $\mathrm{dTB} / \mathrm{sTB}$ levels on POD 3 was significantly different. The dTB and dDB levels were significantly increased in patients with clinically significant bile leakage compared with patients without clinically significant bile leakage on PODs 2, 3, and 5 . There was no hyperbilirubinemia in patients with clinically significant bile leakage. However, there were 2 patients with sTB levels over $5 \mathrm{mg} / \mathrm{dL}$ at POD 5 in patients without clinically significant bile leakage (one who had post-operative liver failure and one who spontaneously improved).

Table 2 shows the ROC analysis that was conducted to determine the best cut-off value for $\mathrm{dTB}$ and $\mathrm{dDB}$ on PODs $1,2,3$, and 5 for clinically significant bile leakage. The AUC of $\mathrm{dDB}$ was higher than that of $\mathrm{dTB}$ on each POD. The AUC of $\mathrm{dDB}$ was $>0.75 \mathrm{mg} / \mathrm{dL}$ on PODs 2,3 , and 5 , and it then increased as the POD increased.

Table 3 shows the predictive power of each cut-off value for clinically significant bile leakage. The $\mathrm{dDB}$ on POD 5 showed the highest accuracy $(0.91)$ and positive predictive value (PPV) (0.67), which was followed by $\mathrm{dTB} / \mathrm{sTB}$ on POD 3 (accuracy, 0.91; PPV, 0.33). Because the PPV for $\mathrm{dDB}$ increased as the POD increased, $\mathrm{dDB}$ was a better predictor of clinically significant bile leakage than dTB. The $\mathrm{dDB}$ on POD 3 showed the highest negative predictive value (NPV) (0.97). The positive likelihood of $\mathrm{dDB}$ increased and the negative likelihood of $\mathrm{dDB}$ decreased as the POD increased.

Figure 1 shows the incidence of clinically significant bile leakage compared with $\mathrm{dTB} / \mathrm{sTB}$ on POD 3 (cut-off, 3 ) and dDB on POD 3 (cut-off, $0.44 \mathrm{mg} / \mathrm{dL}$ ). Among pa- tients with $\mathrm{dTB} / \mathrm{sTB} \leq 3.0$ on POD $3(n=226), 4.8 \%(n=$ 11) developed clinically significant bile leakage. Conversely, among patients with $\mathrm{dTB} / \mathrm{sTB}>3.0$ on POD 3 $(n=15), 46.7 \%(n=7)$ did not develop clinically significant bile leakage. Patients with a $\mathrm{dDB}$ on POD 3 that was $>0.44 \mathrm{mg} / \mathrm{dL}$ had a significantly higher incidence of clinically significant bile leakage than those with a $\mathrm{dDB}$ on POD 3 of $\leq 0.44 \mathrm{mg} / \mathrm{dL}$ in patients with and without dTB/ sTB $>3$ on POD 3 . Although patients with $\mathrm{dTB} / \mathrm{sTB} \leq 3$ on POD 3, 19.1\% patients had clinically significant bile leakage when $\mathrm{dDB}$ was $>0.44 \mathrm{mg} / \mathrm{dL}$ on POD 3 . However, patients who had dTB/sTB $>3$ on POD 3 showed no clinically significant bile leakage when $\mathrm{dDB}$ was $\leq 0.44 \mathrm{mg} / \mathrm{dL}$ on POD 3. Table 4 shows the clinical outcome of 7 patients with $\mathrm{dDB} \leq 0.44 \mathrm{mg} / \mathrm{dL}$ and $\mathrm{dTB} / \mathrm{sTB} \leq 3.0$ on POD 3 and 4 patients with $\mathrm{dDB}>0.44 \mathrm{mg} / \mathrm{dL}$ and $\mathrm{dTB} / \mathrm{sTB}$ $\leq 3.0$ on POD 3 . After removing the drain, clinically significant bile leakage was found in 3 of 7 patients with $\mathrm{dDB}$ $\leq 0.44 \mathrm{mg} / \mathrm{dL}$ and $\mathrm{dTB} / \mathrm{sTB} \leq 3.0$ on POD 3 because of increased inflammatory results. Thus, percutaneous drainage was required. On the basis of patients with $\mathrm{dDB}>0.44$ $\mathrm{mg} / \mathrm{dL}$ and $\mathrm{dTB} / \mathrm{sTB} \leq 3.0$ on POD 3 , it is possible to respond relatively quickly to clinically significant bile leakage. Three of these 4 patients were discharged within 1 month.

\section{Discussion}

In this study, the predictive power of $\mathrm{dDB}$ to detect clinically significant bile leakage after hepatectomy was examined. PPV of dDB increased as the POD increased, and $\mathrm{dDB}$ on POD 3 and POD 5 were useful for predicting clinically significant bile leakage. The definition of ISGPS, which includes $\mathrm{dTB} / \mathrm{sTB}>3$ on POD 3 , has already been established, but the combination of $\mathrm{dTB} / \mathrm{sTB}$ with $\mathrm{dDB}$ is useful for detecting clinically significant bile leakage.

The incidence of clinically significant bile leakage was $6.2 \%$ in this study, which is similar to the previous reports, which ranged from $4.8 \%$ to $10.2 \%$ [11-15]. Several reports have focused on bile leakage after liver resection, but there are no reports that focused on $\mathrm{dDB}$ [11-20]. Our report is the first to investigate the predictive significance of $\mathrm{dDB}$ for clinically significant bile leakage. dTB is sometimes elevated and affected by minor bleeding from the cut surface of the liver, and this haemolysis will affect dTB but not dDB. Direct bilirubin is conjugated bilirubin that originates from hepatocytes, and $\mathrm{dDB}$ also indicates if there is no post-operative liver failure. Many hepatobiliary centres may not use routine drainage after
36

Eur Surg Res 2022;63:33-39 DOI: $10.1159 / 000518267$
Harimoto/Araki/Yamanaka/Hagiwara/ Ishii/Tsukagoshi/Watanabe/Shirabe 
Table 3. Predictive power of drainage factors for clinically significant bile leakage

\begin{tabular}{lllllllrrrrr}
\hline Variables & $N$ & $\begin{array}{l}\text { Cut-off value, } \\
\text { mg/dL }\end{array}$ & Sensitivity & Specificity & Accuracy & PPV & NPV & LR+ & LR- & $p$ value \\
\hline dTB/sTB (3POD) & 241 & 3 & 0.31 & 0.96 & 0.91 & 0.33 & 0.95 & 7.75 & 0.72 & $<0.01$ \\
dTB (1POD) & 257 & 0.90 & 0.50 & 0.58 & 0.57 & 0.07 & 0.94 & 1.19 & 0.86 & 0.61 \\
dTB (2POD) & 257 & 1.60 & 0.50 & 0.83 & 0.80 & 0.16 & 0.96 & 2.94 & 0.60 & $<0.01$ \\
dTB (3POD) & 241 & 2.10 & 0.56 & 0.74 & 0.73 & 0.13 & 0.96 & 2.15 & 0.59 & 0.02 \\
dTB (5POD) & 157 & 4.10 & 0.40 & 0.86 & 0.82 & 0.24 & 0.93 & 2.85 & 0.70 & 0.02 \\
dDB (1POD) & 257 & 0.34 & 0.50 & 0.81 & 0.79 & 0.15 & 0.96 & 2.63 & 0.62 & $<0.01$ \\
dDB (2POD) & 257 & 0.21 & 0.63 & 0.77 & 0.75 & 0.15 & 0.96 & 2.74 & 0.48 & $<0.01$ \\
dDB (3POD) & 241 & 0.44 & 0.56 & 0.91 & 0.89 & 0.31 & 0.97 & 6.22 & 0.48 & $<0.01$ \\
dDB (5POD) & 157 & 1.35 & 0.53 & 0.97 & 0.93 & 0.67 & 0.95 & 17.67 & 0.48 & 0.01 \\
\hline
\end{tabular}

AUC, area under the curve; LR+, positive likelihood; PPV, positive predictive value; NPV, negative predictive value; LR-, negative likelihood; dTB, total bilirubin level in drainage fluid; sTB, serum total bilirubin level; dDB, direct bilirubin level in drainage fluid; POD, post-operative day.

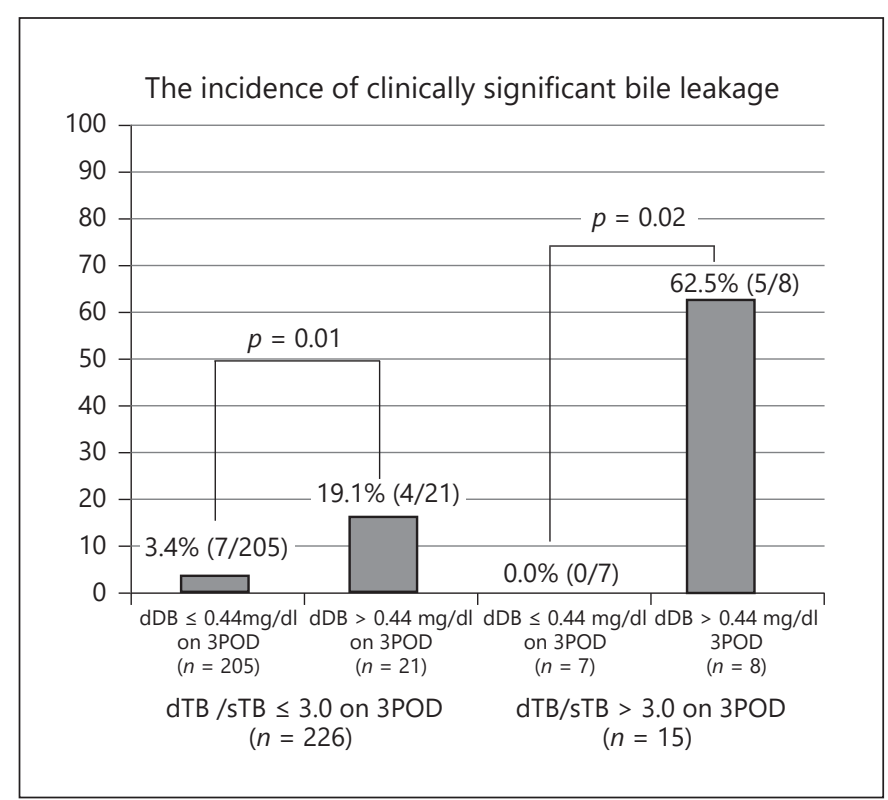

Fig. 1. Incidence of severe bile leakage after hepatectomy. Although patients had $\mathrm{dTB} / \mathrm{sTB} \leq 3$ at POD $3,19.1 \%$ of these patients had bile leakage when $\mathrm{dDB}$ was $>0.4 \mathrm{mg} / \mathrm{dL}$ on POD 3 . However, patients with $\mathrm{dTB} / \mathrm{sTB}>3$ at POD 3 showed no bile leakage when $\mathrm{dDB}$ was $\leq 0.44 \mathrm{mg} / \mathrm{dL}$ on POD 3. $\mathrm{dTB}$, drainage fluid total bilirubin level; sTB, serum total bilirubin level; $\mathrm{dDB}$, drainage fluid direct bilirubin level; POD, post-operative day.

standard hepatectomy, but insertion after surgery has a certain risk and causes stress. Detecting clinically significant bile leakage at an early stage can lead to a relatively quick response, and thus, early improvement is possible.

The ISGLS definition and severity grading of bile leakage after hepatectomy are correlated with clinical out-
Table 4. Clinical outcome of patients with clinically significant bile leakage

\begin{tabular}{lllll}
\hline Case & $\begin{array}{l}\text { PODs when } \\
\text { bile leakage } \\
\text { was found }\end{array}$ & Intervention & $\begin{array}{l}\text { Duration } \\
\text { of drainage, } \\
\text { days }\end{array}$ & $\begin{array}{l}\text { Hospital } \\
\text { stay, } \\
\text { days }\end{array}$ \\
\hline $\mathrm{dDB} \leq 0.44 \mathrm{mg} / \mathrm{dL}$ and dTB/sTB $\leq 3.0$ on 3 POD & \\
1 & 7POD & PAD & - & 77 \\
2 & 9POD & PAD & - & 38 \\
3 & 10POD & PAD & - & 65 \\
4 & 4POD & Drain exchange & 25 & 37 \\
5 & 7POD & Drain exchange & 23 & 60 \\
6 & 7POD & EBD & 30 & 62 \\
7 & 11POD & Drain exchange & 43 & 76 \\
\hline $\mathrm{dDB}>0.44 \mathrm{mg} / \mathrm{dL}$ and dTB/sTB $\leq 3.0$ on $3 \mathrm{POD}$ & \\
1 & 4POD & Drain exchange & 14 & 21 \\
2 & 5POD & ENBD & 19 & 22 \\
3 & 5POD & ENBD & 25 & 28 \\
4 & 6POD & ENBD & 66 & 71 \\
\hline
\end{tabular}

dTB, total bilirubin level in drainage fluid; sTB, serum total bilirubin level; $\mathrm{dDB}$, direct bilirubin level in drainage fluid; POD, post-operative day; PAD, percutaneous abscess drainage; ENBD, endoscopic naso-biliary drainage.

comes [4, 5]. Rahbari et al. [5] reported that dTB $\geq 2.4 \mathrm{mg} /$ $\mathrm{dL}$ on POD 2 had the highest AUC (0.82). Taguchi et al. [16] also reported that dTB $\geq 3.7 \mathrm{mg} / \mathrm{dL}$ on POD 3 had the highest AUC (0.746). Donadon et al. [17] reported that the AUC on POD 7 was the highest (0.813). Yamazaki et al. [18] reported that $\mathrm{dTB} \geq 3.01 \mathrm{mg} / \mathrm{dL}$ on POD 3 had the highest accuracy (0.836). Our study showed that the AUC of $\mathrm{dDB}$ on POD 3 was 0.794 and that on POD 5 was 0.809 . 
The accuracy of $\mathrm{dDB}$ had the highest predictive value for severe bile leakage compared with dTB. The NPV for predicting bile leakage is important because the drain will be removed within the cut-off values. In this study, the NPV for $\mathrm{dDB}$ on POD 3 was 0.97 , which was the highest value. The predictive power of $\mathrm{dTB} / \mathrm{sTB}>3$ on POD 3 also showed significant accuracy (0.91) and NPV (0.95). Additionally, even if $\mathrm{dTB} / \mathrm{sTB}>3$ on POD 3 was positive, we could assume that there was no severe bile leakage if $\mathrm{ADB}$ on POD 3 was $<0.44 \mathrm{mg} / \mathrm{dL}$. The combination of $\mathrm{dTB} / \mathrm{sTB}$ and $\mathrm{dDB}$ on POD 3 is useful for predicting severe bile leakage.

The timing for removing the drain is controversial. Tanaka et al. [19] reported that the positivity rate for bacteriologic cultures of drainage fluid on POD 7 or later was $71.4 \%$, and early removal of the drain was recommended. Detecting severe bile leakage on an early POD is useful for preventing an infection in the drainage fluid. However, bile leakage after drain removal was expected to be a disadvantage of early drain removal. Figure 1 shows that on the basis of $\mathrm{dTB} / \mathrm{sTB}>3$ on POD 3 , false-negative results $(46.7 \%, 7 / 15)$ can be detected using $\mathrm{dDB} \leq 0.44 \mathrm{mg} / \mathrm{dL}$ on POD 3. However, false-positive results $(1.8 \%, 4 / 226)$ can be detected using $\mathrm{dDB}>0.44 \mathrm{mg} / \mathrm{dL}$ on POD 3 . We determined the appropriateness of drain removal on POD 3 using $\mathrm{dTB}$ and $\mathrm{dDB}$. If there is a concern, the patient will be reassessed on POD 5. Kaibori et al. [20] reported lateonset bile leakage after hepatic resection, but the incidence of late-onset bile leakage was rare $(1.78 \% ; 18 / 1,004)$. Although late-onset bile leakage can cause serious complications, there were no patients with late-onset bile leakage.

This study has several limitations. Firstly, the sample size was small. Further studies with a larger, multi-institutional sample size using a training set and validation set will lead to a definitive conclusion. Secondly, the timing for removing the drain depended on the attending doctor. Thus, a prospective protocol is required.

This retrospective analysis showed that $\mathrm{dDB}$ on $\mathrm{POD}$ 3 was useful for predicting clinically significant bile leakage in patients who were undergoing hepatectomy for he- patic malignancy. Measurement of both the $\mathrm{dDB}$ and $\mathrm{dTB}$, which are easy to perform, can be used to effectively predict clinically significant bile leakage.

\section{Acknowledgements}

We thank Mark Abramovitz, PhD, and Jodi Smith, PhD ELS from Edanz (https://www.edanz.com/ac), for editing a draft of this manuscript.

\section{Statement of Ethics}

The study protocol was reviewed and approved by our Institutional Ethics Committee (approval number: HS2019-306). Written informed consent was obtained from the participants (or their parent/legal guardian/next of kin) to participate in the study.

\section{Conflict of Interest Statement}

The authors have no conflicts of interest to declare.

\section{Funding Sources}

The authors have no grants and financial support.

\section{Author Contributions}

Study conception and design: N. Harimoto and K. Shirabe. Acquisition of data: T. Yamanaka, K. Hagiwara, and N. Ishii. Analysis and interpretation of data: M. Tsukagoshi and A. Watanabe. Drafting of manuscript: N. Harimoto. Critical revision: K. Shirabe.

\section{Data Availability Statement}

All data that were generated or analysed during this study are included in this article. Further enquiries can be directed to the corresponding author.

\section{References}

1 Kamiyama T, Nakanishi K, Yokoo $\mathrm{H}$, Kamachi H, Tahara M, Yamashita K, et al. Perioperative management of hepatic resection toward zero mortality and morbidity: analysis of 793 consecutive cases in a single institution. J Am Coll Surg. 2010;211(4): 443-9.

2 Fan ST, Lo CM, Liu CL, Lam CM, Yuen WK, Yeung C, et al. Hepatectomy for hepatocellu- lar carcinoma: toward zero hospital deaths. Ann Surg. 1999;229(3):322-30.

3 Harimoto N, Muranushi R, Hoshino K, Yamanaka T, Hagiwara K, Ishii N, et al. Albumin-indocyanine green evaluation (ALICE) grade predicts bile leakage after hepatic resection. Surg Today. 2020;50(8):849-54.

4 Koch M, Garden OJ, Padbury R, Rahbari NN, Adam R, Capussotti L, et al. Bile leakage after hepatobiliary and pancreatic surgery: a definition and grading of severity by the International study group of liver surgery. Surgery. 2011;149(5):680-8.

5 Rahbari NN, Elbers H, Koch M, Kirchberg J, Dutlu M, Mehrabi A, et al. Bilirubin level in the drainage fluid is an early and independent predictor of clinically relevant bile leakage after hepatic resection. Surgery. 2012;152(5):821-31.
38

Eur Surg Res 2022;63:33-39

DOI: $10.1159 / 000518267$
Harimoto/Araki/Yamanaka/Hagiwara/ Ishii/Tsukagoshi/Watanabe/Shirabe 
6 Kokudo T, Hasegawa K, Amikura K, Uldry E, Shirata C, Yamaguchi T, et al. Assessment of preoperative liver function in patients with hepatocellular carcinoma: the albumin-indocyanine green evaluation (ALICE) grade. PLoS One. 2016;11(7):e0159530.

7 Taketomi A, Kitagawa D, Itoh S, Harimoto N, Yamashita Y, Gion T, et al. Trends in morbidity and mortality after hepatic resection for hepatocellular carcinoma: an institute's experience with 625 patients. J Am Coll Surg. 2007; 204(4):580-7.

8 Harimoto N, Shirabe K, Ikegami T, Yoshizumi T, Maeda T, Kajiyama K, et al. Postoperative complications are predictive of poor prognosis in hepatocellular carcinoma. J Surg Res. 2015;199(2):470-7.

9 Araki K, Harimoto N, Kubo N, Watanabe A, Igarashi $\mathrm{T}$, Tsukagoshi $\mathrm{M}$, et al. Functional liver volumetry using Gd-EOB-DTPA-enhanced magnetic resonance imaging (MRI) predicts post-hepatectomy liver failure in resection of more than one segment. HPB. 2019; S1365(19):20687-2.

10 Rahbari NN, Koch M, Mehrabi A, Weidmann K, Motschall E, Kahlert C, et al. Portal triad clamping versus vascular exclusion for vascular control during hepatic resection: a systematic review and meta-analysis. J Gastrointest Surg. 2009;13:558-68.
11 Ishii H, Ochiai T, Murayama Y, Komatsu S, Shiozaki A, Kuriu Y, et al. Risk factors and management of postoperative bile leakage after hepatectomy without bilioenteric anastomosis. Dig Surg. 2011;28(3):198-204.

12 Sadamori H, Yagi T, Shinoura S, Umeda Y, Yoshida R, Satoh D, et al. Risk factors for major morbidity after liver resection for hepatocellular carcinoma. Br J Surg. 2013;100(1):122-9.

13 Nakagawa K, Tanaka K, Nojiri K, Sawada Y, Kumamoto T, Ueda M, et al. Predictive factors for bile leakage after hepatectomy for hepatic tumors: a retrospective multicenter study with 631 cases at Yokohama clinical oncology group (YCOG). J Hepatobiliary Pancreat Sci. 2017;24(1):33-41.

14 Yamashita Y, Hamatsu T, Rikimaru T, Tanaka S, Shirabe K, Shimada M, et al. Bile leakage after hepatic resection. Ann Surg. 2001; 233(1):45-50

15 Panaro F, Hacina L, Bouyabrine H, Al-Hashmi AW, Herrero A, Navarro F. Risk factors for postoperative bile leakage: a retrospective single-center analysis of 411 hepatectomies. Hepatobiliary Pancreat Dis Int. 2016;15(1): 81-6.
16 Taguchi Y, Ebata T, Yokoyama Y, Igami T, Sugawara G, Kokuryo T, et al. The determination of bile leakage in complex hepatectomy based on the guidelines of the International study group of liver surgery. World J Surg. 2014;38(1):168-76.

17 Donadon M, Costa G, Cimino M, Procopio F, Del Fabbro D, Palmisano A, et al. Diagnosis and management of bile leaks after hepatectomy: results of a prospective analysis of 475 hepatectomies. World J Surg. 2016;40(1): $172-81$.

18 Yamazaki S, Takayama T, Moriguchi M, Mitsuka Y, Okada S, Midorikawa Y, et al. Criteria for drain removal following liver resection. $\mathrm{Br}$ J Surg. 2012;99(11):1584-90.

19 Tanaka K, Kumamoto T, Nojiri K, Takeda K, Endo I. The effectiveness and appropriate management of abdominal drains in patients undergoing elective liver resection: a retrospective analysis and prospective case series. Surg Today. 2013;43:372-80.

20 Kaibori M, Shimizu J, Hayashi M, Nakai T, Ishizaki M, Matsui $\mathrm{K}$, et al. Late-onset bile leakage after hepatic resection. Surgery. 2015; 157(1):37-44. 